\title{
An efficient dual to ratio-cum-product estimator for the population mean in stratified random sampling
}

\author{
Rohini Yadav \\ Department of Statistics, Amity Institute of Applied Sciences, Amity University, Noida, U.P., India
}

Available online at: www.isroset.org

Received: 13/Dec/ 2017, Revised: 24/Dec/2017, Accepted: 31/Dec/2017, Online: 30/Apr/2018

\begin{abstract}
This paper deals with the problem of estimating the population mean of the study variable y with the help of the auxiliary variables under stratified random sampling. The estimator of dual to ratio-cum-product estimator for the population mean of the study variable has been suggested. The properties of the suggested estimator have been studied under large sample approximation. It has been shown that the suggested estimator is more efficient than other considered estimators. To judge the merits of the proposed estimator, an empirical study is given in the support of the present study.
\end{abstract}

Keywords- Study variable, auxiliary variable, stratified random sampling, dual to ratio estimator, dual to product estimator, bias and mean squared error.

\section{INTRODUCTION}

It is well known fact that the supplementary or auxiliary information always increases the precision of the estimators for the population parameters of the study variable. Ratio, product, regression and ratio-cum-product type of estimators are good examples in this context. [1] proposed the ratio estimator assuming that the study variable (y) and auxiliary variable $(\mathrm{x})$ are positively correlated and the population mean of the auxiliary variable is known. But when the study variable (y) and the auxiliary variable (x) are negatively correlated then the ratio estimator does not perform well. In that situation, the product estimator envisaged by [2] is used. Sometimes it is observed that the study variable has positive as well as negative correlation with two different variables at a time. This observation encourages using both positively and negatively correlated variables in the study.

Many authors [3], [4], [5], [6], [7] and [8] have proposed ratio type estimators and ratio-cum-product estimators of the finite population mean $\bar{Y}$ of the study variable using the values of known parameters of the auxiliary variables in simple random sampling.

First [9] proposed dual to ratio estimator and [10] suggested dual to product estimator for the population mean using transformation on auxiliary variable under simple random sampling.
Due to some shortcomings under simple random sampling, many authors [11], [12], [13], [14] and [15] have defined ratio estimators and ratio-cum-product estimators under stratified random sampling which perform better than usual ratio and product estimators in simple random sampling under certain limitations.

Motivated by them, an attempt is made to develop an efficient dual to ratio-cum-product estimator of the population mean of the study variable using the knowledge of coefficient of variation of the auxiliary variable under stratified random sampling.

Consider the population of size $\mathrm{N}$ is equally divided into $\mathrm{L}$ strata with $\mathrm{N}_{\mathrm{h}}$ elements in the $\mathrm{h}^{\text {th }}$ stratum such that $\mathrm{N}=\sum_{\mathrm{h}=1}^{\mathrm{L}} \mathrm{N}_{\mathrm{h}}$. Let $\mathrm{y}$ be the study variable and $\mathrm{x}$ and $\mathrm{z}$ be two auxiliary variables assuming values $y_{h i}, x_{h i}$ and $z_{h i}$ for the $\mathrm{i}^{\text {th }}$ unit in $\mathrm{h}^{\text {th }}$ stratum. Let $\mathrm{n}_{\mathrm{h}}$ be the size of the sample drawn from $h^{\text {th }}$ stratum of size $N_{h}$ by using simple random sampling without replacement (SRSWOR) such that sample size $n=\sum_{h=1}^{L} n_{h}$. We define 
$\bar{Y}_{h}=\frac{1}{N_{h}} \sum_{i=1}^{N_{h}} y_{h i}: \quad h^{t h}$ stratum mean for the study

variate $\mathrm{y}$

$\bar{X}_{h}=\frac{1}{N_{h}} \sum_{i=1}^{N_{h}} x_{h i}: \quad h^{\text {th }}$ stratum mean for the study

variate $\mathrm{x}$

$\bar{Z}_{h}=\frac{1}{N_{h}} \sum_{i=1}^{N_{h}} z_{h i}: \quad h^{t h}$ stratum mean for the study

variate $\mathrm{z}$

$\bar{Y}=\frac{1}{N} \sum_{h=1}^{L} \sum_{i=1}^{N_{h}} y_{h i}=\frac{1}{N} \sum_{h=1}^{L} N_{h} \bar{Y}_{h}=\sum_{h=1}^{L} W_{h} \bar{Y}_{h}:$

population mean of the study variate $y$

$\bar{X}=\frac{1}{N} \sum_{h=1}^{L} \sum_{i=1}^{N_{h}} x_{h i}=\frac{1}{N} \sum_{h=1}^{L} W_{h} \bar{X}_{h}$

population mean of the auxiliary variate $\mathrm{x}$

$\bar{Z}=\frac{1}{N} \sum_{h=1}^{L} \sum_{i=1}^{N_{h}} z_{h i}=\frac{1}{N} \sum_{h=1}^{L} W_{h} \bar{Z}_{h}$

population mean of the study variate $\mathrm{z}$

$\bar{y}_{h}=\frac{1}{n_{h}} \sum_{i=1}^{n_{h}} y_{h i}:$ sample mean of the study variate $\mathrm{y}$ for

$h^{\text {th }}$ stratum

$\bar{x}_{h}=\frac{1}{n_{h}} \sum_{i=1}^{n_{h}} x_{h i}:$ sample mean of the auxiliary variate $\mathrm{x}$ for

$h^{\text {th }}$ stratum

$\bar{z}_{h}=\frac{1}{n_{h}} \sum_{i=1}^{n_{h}} z_{h i}:$ sample mean of the auxiliary variate $\mathrm{z}$ for

$h^{\text {th }}$ stratum

$W_{h}=\frac{N_{h}}{N}:$ stratum weight of $h^{\text {th }}$ stratum.
On the line of [1], [16] defined the classical combined ratio estimator for the population mean of the study variable $y$ under stratified random sampling as

$\bar{y}_{R C}=\bar{y}_{s t}\left(\frac{\bar{X}}{\bar{x}_{s t}}\right)$

where $\bar{y}_{s t}=\sum_{h=1}^{L} W_{h} \bar{y}_{h}$ and $\bar{x}_{s t}=\sum_{h=1}^{L} W_{h} \bar{x}_{h}$

Here it is assumed that the study variable $y$ and auxiliary variable $\mathrm{x}$ are positively correlated.

The combined product estimator of the population mean of the study variable $y$ is defined as

$\bar{y}_{P C}=\bar{y}_{s t}\left(\frac{\bar{z}_{s t}}{\bar{Z}}\right)$

where $\bar{z}_{s t}=\sum_{h=1}^{L} W_{h} \bar{z}_{h}$

Using the concept of dual to ratio and dual to product estimators, [17] proposed dual to ratio and dual to product estimators of the population mean of the study variable $y$ under stratified random sampling which are defined as

$\bar{y}_{R C}^{*}=\bar{y}_{s t}\left(\frac{\bar{x}_{s t}^{*}}{\bar{X}}\right)$

$\bar{y}_{P C}^{*}=\bar{y}_{s t}\left(\frac{\bar{Z}}{\bar{z}_{s t}^{*}}\right)$

where $\bar{x}_{s t}^{*}=\sum_{h=1}^{L} W_{h} \bar{x}_{h}^{*} \quad$ and $\quad \bar{z}_{s t}^{*}=\sum_{h=1}^{L} W_{h} \bar{z}_{h}^{*}$

Here, transformations based on [9] are

$\bar{x}_{h}^{*}=\left(\frac{N_{h} \bar{X}_{h}-n_{h} \bar{x}_{h}}{N_{h}-n_{h}}\right)$ and $\bar{z}_{h}^{*}=\left(\frac{N_{h} \bar{Z}_{h}-n_{h} \bar{z}_{h}}{N_{h}-n_{h}}\right)$ 
Later on, [18] proposed improved dual to ratio and dual to product estimators of the study variable y using information based on auxiliary variables $\mathrm{x}$ and $\mathrm{z}$ under stratified random sampling defined as

$\bar{y}_{\mathrm{Re}}^{* s t}=\bar{y}_{s t} \exp \left(\frac{\bar{x}_{s t}^{*}-\bar{X}}{\bar{x}_{s t}^{*}+\bar{X}}\right)=\bar{y}_{s t} \exp \left[\frac{\sum_{h=1}^{L} W_{h}\left(\bar{x}_{h}^{*}-\bar{X}_{h}\right)}{\sum_{h=1}^{L} W_{h}\left(\bar{x}_{h}^{*}+\bar{X}_{h}\right)}\right]$

and

$$
\bar{y}_{p \mathrm{e}}^{* s t}=\bar{y}_{s t} \exp \left(\frac{\bar{Z}-\bar{z}_{s t}^{*}}{\bar{Z}+\bar{z}_{s t}^{*}}\right)=\bar{y}_{s t} \exp \left[\frac{\sum_{h=1}^{L} W_{h}\left(\bar{Z}_{h}-\bar{z}_{h}^{*}\right)}{\sum_{h=1}^{L} W_{h}\left(\bar{Z}_{h}+\bar{z}_{h}^{*}\right)}\right]
$$

The mean squared errors (MSE) of the combined ratio estimator $\bar{y}_{R C}$, the combined product estimator $\bar{y}_{P C}$, estimators due to [17] $\bar{y}_{R C}^{*}$ and $\bar{y}_{P C}^{*}$ and the estimators due to [18] $\bar{y}_{\mathrm{Re}}^{* s t}$ and $\bar{y}_{P e}^{* s t}$ defined in (1), (2), (3), (4), (5) and (6) upto the first order of approximation are respectively given by

$$
\begin{aligned}
& \operatorname{MSE}\left(\bar{y}_{R C}\right)=\sum_{h=1}^{L} W_{h}^{2} \gamma_{h}\left(S_{y h}^{2}+R_{1}^{2} S_{x h}^{2}-2 R_{1} S_{y x h}\right) \\
& \operatorname{MSE}\left(\bar{y}_{P C}\right)=\sum_{h=1}^{L} W_{h}^{2} \gamma_{h}\left(S_{y h}^{2}+R_{2}^{2} S_{z h}^{2}+2 R_{2} S_{y z h}\right) \\
& \operatorname{MSE}\left(\bar{y}_{R C}^{*}\right)=\sum_{h=1}^{L} W_{h}^{2} \gamma_{h}\left(S_{y h}^{2}+R_{1}^{2} g_{h}^{2} S_{x h}^{2}-2 R_{1} g_{h} S_{y x h}\right)
\end{aligned}
$$

$$
\operatorname{MSE}\left(\bar{y}_{P C}^{*}\right)=\sum_{h=1}^{L} W_{h}^{2} \gamma_{h}\left(S_{y h}^{2}+R_{2}^{2} g_{h}^{2} S_{z h}^{2}+2 R_{2} g_{h} S_{y z h}\right)
$$

$$
\operatorname{MSE}\left(\bar{y}_{\mathrm{Re}}^{* s t}\right)=\sum_{h=1}^{L} W_{h}^{2} \gamma_{h}\left(S_{y h}^{2}+\frac{R_{1}^{2}}{4} g_{h}^{2} S_{x h}^{2}-R_{1} g_{h} S_{y x h}\right)
$$

$$
\operatorname{MSE}\left(\bar{y}_{P \mathrm{e}}^{* s t}\right)=\sum_{h=1}^{L} W_{h}^{2} \gamma_{h}\left(S_{y h}^{2}+\frac{R_{2}^{2}}{4} g_{h}^{2} S_{z h}^{2}+R_{2} g_{h} S_{y z h}\right)
$$

where $\quad \mathrm{g}_{\mathrm{h}}=\frac{n_{n}}{N_{h}-n_{n}}, \quad \gamma_{h}=\left(\frac{1}{n_{h}}-\frac{1}{N_{h}}\right)$,

$$
\begin{aligned}
& R_{1}=\frac{\bar{Y}}{\bar{X}}, \quad R_{2}=\frac{\bar{Y}}{\bar{Z}}, \\
& S_{y h}^{2}=\frac{1}{N_{h}-1} \sum_{i=1}^{N_{h}}\left(y_{h i}-\bar{Y}_{h}\right)^{2} \text {, } \\
& S_{x h}^{2}=\frac{1}{N_{h}-1} \sum_{i=1}^{N_{h}}\left(x_{h i}-\bar{X}_{h}\right)^{2} \text {, } \\
& S_{z h}^{2}=\frac{1}{N_{h}-1} \sum_{i=1}^{N_{h}}\left(z_{h i}-\bar{Z}_{h}\right)^{2} \text {, } \\
& S_{y x h}=\frac{1}{N_{h}-1} \sum_{i=1}^{N_{h}}\left(y_{h i}-\bar{Y}_{h}\right)\left(x_{h i}-\bar{X}_{h}\right) \text {, } \\
& S_{y z h}=\frac{1}{N_{h}-1} \sum_{i=1}^{N_{h}}\left(y_{h i}-\bar{Y}_{h}\right)\left(z_{h i}-\bar{Z}_{h}\right) \\
& S_{x z h}=\frac{1}{N_{h}-1} \sum_{i=1}^{N_{h}}\left(x_{h i}-\bar{X}_{h}\right)\left(z_{h i}-\bar{Z}_{h}\right) .
\end{aligned}
$$

\section{THE SUGGESTED ESTIMATOR}

The modified dual to ratio-cum-product estimator has been proposed for the population mean of the study variable $y$ using known value of the coefficient of variation of the auxiliary variable $\mathrm{x}$ under stratified random sampling defined as 


$$
t_{s t}^{*}=\bar{y}_{s t}\left[\alpha\left\{\frac{\sum_{h=1}^{L} W_{h}\left(\bar{x}_{h}^{*}+C_{x h}\right)}{\sum_{h=1}^{L} W_{h}\left(\bar{X}_{h}+C_{x h}\right)}\right\}+(1-\alpha) \exp \left\{\frac{\sum_{h=1}^{L} W_{h}\left\{\bar{Z}_{h}-\bar{z}_{h}^{*}\right\}}{\sum_{h=1}^{L} W_{h}\left\{\bar{Z}_{h}+\bar{z}_{h}^{*}\right\}}\right\}\right]
$$

where $\alpha$ is a suitable constant to be determined such that MSE of the proposed estimator is minimum.

Using transformation on $\bar{x}_{h}^{*}$ and $\bar{z}_{h}^{*}$ of the auxiliary variables $\mathrm{x}$ and $\mathrm{z}$, the suggested estimator $t_{s t}^{*}$ in (13) can be written as

$$
\begin{aligned}
t_{s t}^{*}=\left(\sum_{h=1}^{L} W_{h} \bar{y}_{h}\right) & {\left[\alpha \frac{\sum_{h=1}^{L} W_{h}\left(\left(\frac{N_{h} \bar{X}_{h}-n_{h} \bar{x}_{h}}{N_{h}-n_{h}}\right)+C_{x h}\right)}{\sum_{h=1}^{L} W_{h}\left(\bar{X}_{h}+C_{x h}\right)}\right\} } \\
& \left.+(1-\alpha) \exp \left\{\frac{\sum_{h=1}^{L} W_{h}\left(\bar{Z}_{h}-\frac{N_{h} \bar{Z}_{h}-n_{h} \bar{z}_{h}}{N_{h}-n_{h}}\right)}{\sum_{h=1}^{L} W_{h}\left(\bar{Z}_{h}+\frac{N_{h} \bar{Z}_{h}-n_{h} \bar{z}_{h}}{N_{h}-n_{h}}\right)}\right\}\right]
\end{aligned}
$$

Consider

$\bar{y}_{h}=\bar{Y}\left(1+e_{0 h}\right), \quad \bar{x}_{h}=\bar{X}_{h}\left(1+e_{1 h}\right) \quad$ and $\quad \bar{z}_{h}=\bar{Z}_{h}\left(1+e_{2 h}\right)$

such that $E\left(e_{0 h}\right)=E\left(e_{1 h}\right)=E\left(e_{2 h}\right)=0$

$E\left(e_{0 h}^{2}\right)=\gamma_{h} C_{y h}^{2}, \quad E\left(e_{1 h}^{2}\right)=\gamma_{h} C_{x h}^{2}, \quad E\left(e_{2 h}^{2}\right)=\gamma_{h} C_{z h}^{2}$,

$E\left(e_{0 h} e_{1 h}\right)=\gamma_{h} \rho_{y x h} C_{y h} C_{x h}=\gamma_{h} \frac{S_{y x h}}{\bar{Y}_{h} \bar{X}_{h}}$

$E\left(e_{1 h} e_{2 h}\right)=\gamma_{h} \rho_{x z h} C_{x h} C_{z h}=\gamma_{h} \frac{S_{x z h}}{\bar{X}_{h} \bar{Z}_{h}}$

and $E\left(e_{0 h} e_{2 h}\right)=\gamma_{h} \rho_{y z h} C_{y h} C_{z h}=\gamma_{h} \frac{S_{y z h}}{\bar{Y}_{h} \bar{Z}_{h}}$.

Expressing (14) in terms of e's, we get

$$
=\bar{Y}\left(1+e_{0}\right)\left[\alpha\left(1-e_{1}\right)+(1-\alpha) \exp \left\{e_{2}\left(1-e_{2}\right)^{-1}\right\}\right]
$$

where $e_{0}=\frac{\sum_{h=1}^{L} W_{h} \bar{Y}_{h} e_{0 h}}{\sum_{h=1}^{L} W_{h} \bar{Y}_{h}}=\frac{\sum_{h=1}^{L} W_{h} \bar{Y}_{h} e_{0 h}}{\bar{Y}}$,

$e_{1}=\frac{\sum_{h=1}^{L} W_{h} g_{h} \bar{X}_{h} e_{1 h}}{\sum_{h=1}^{L} W_{h}\left\{\bar{X}_{h}+C_{x h}\right\}}=\frac{\sum_{h=1}^{L} W_{h} g_{h} \bar{X}_{h} e_{1 h}}{\bar{X}_{1 h}}$,

$e_{2}=\frac{1}{2} \frac{\sum_{h=1}^{L} W_{h} g_{h} \bar{Z}_{h} e_{2 h}}{\sum_{h=1}^{L} W_{h} \bar{Z}_{h}}=\frac{\sum_{h=1}^{L} W_{h} g_{h} \bar{Z}_{h} e_{2 h}}{\bar{Z}_{1 h}}$

and $\quad \mathrm{g}_{\mathrm{h}}=\frac{n_{n}}{N_{h}-n_{n}}$

such that

$E\left(e_{0}\right)=E\left(e_{1}\right)=E\left(e_{2}\right)=0$

and

$$
E\left(e_{0}^{2}\right)=\frac{1}{\bar{Y}^{2}} \sum_{h=1}^{L} W_{h}^{2} \gamma_{h} S_{y h}^{2},
$$

$E\left(e_{1}^{2}\right)=\frac{1}{\bar{X}_{1 h}^{2}} \sum_{h=1}^{L} W_{h}^{2} \gamma_{h} g_{h}^{2} S_{x h}^{2}$,

$E\left(e_{2}^{2}\right)=\frac{1}{\bar{Z}_{1 h}^{2}} \sum_{h=1}^{L} W_{h}^{2} \gamma_{h} g_{h}^{2} S_{z h}^{2}$,

$E\left(e_{0} e_{1}\right)=\frac{1}{\bar{Y} \bar{X}_{1 h}} \sum_{h=1}^{L} W_{h}^{2} \gamma_{h} g_{h} S_{y x h}$,

$E\left(e_{1} e_{2}\right)=\frac{1}{\bar{X}_{1 h} \bar{Z}_{1 h}} \sum_{h=1}^{L} W_{h}^{2} \gamma_{h} g_{h}^{2} S_{x z h}$,

$E\left(e_{0} e_{2}\right)=\frac{1}{\bar{Y} \bar{Z}_{1 h}} \sum_{h=1}^{L} W_{h}^{2} \gamma_{h} g_{h} S_{y z h}$

To the first degree of approximation, the bias and mean squared error of the suggested estimator $t_{s t}^{*}$ are given by 


$$
\begin{array}{rlrl}
B\left(t_{s t}^{*}\right)=\sum_{h=1}^{L} W_{h}^{2} \gamma_{h} g_{h}\left[\frac{1}{\bar{Z}_{1 h}}\left(\frac{3}{2} g_{h} R_{13} S_{z h}^{2}+S_{y z h}\right)\right. & M=\sum_{h=1}^{L} W_{h}^{2} \gamma_{h} S_{y h}^{2} \\
\left.-\frac{\alpha}{\bar{Z}_{1 h}}\left(\frac{3}{2} g_{h} R_{13} S_{z h}^{2}+S_{y z h}+\frac{\bar{Z}_{1 h}}{\bar{X}_{1 h}} S_{y x h}\right)\right] & P=2 \frac{A}{B} \sum_{h=1}^{L} W_{h}^{2} \gamma_{h} g_{h} R_{12} S_{y x h} \\
(15) & Q=\frac{A^{2}}{B^{2}} \sum_{h=1}^{L} W_{h}^{2} \gamma_{h} g_{h}^{2} R_{12}^{2} S_{x h}^{2} \\
M S E\left(t_{s t}^{*}\right)=\sum_{h=1}^{L} W_{h}^{2} \gamma_{h}\left[S_{y h}^{2}-\alpha\left\{2 g_{h} R_{12} S_{y x h}-\alpha g_{h}^{2} R_{12}^{2} S_{x h}^{2}\right\}+(1-\alpha)\right. & R=\left(1-\frac{A}{B}\right)^{2} \sum_{h=1}^{L} W_{h}^{2} \gamma_{h} g_{h}^{2} R_{13}^{2} S_{z h}^{2} \\
\left.\left\{(1-\alpha) g_{h}^{2} R_{13}^{2} S_{z h}^{2}+2 g_{h} R_{13} S_{y z h}-2 \alpha g_{h}^{2} R_{12} R_{13} S_{x z h}\right\}\right] & (16) & S=2\left(1-\frac{A}{B}\right) \sum_{h=1}^{L} W_{h}^{2} \gamma_{h} g_{h} R_{13} S_{y z h} \\
\text { where } & R_{12}=\frac{\bar{Y}}{\sum_{h=1}^{L} W_{h}\left\{\bar{X}_{h}+\beta_{2 h}(x)\right\}}=\frac{A}{\bar{X}_{1 h}} & T & =2 \frac{A}{B}\left(1-\frac{A}{B}\right) \sum_{h=1}^{L} W_{h}^{2} \gamma_{h} g_{h}^{2} R_{12} R_{13} S_{x z h} .
\end{array}
$$

$$
\text { and } \quad R_{13}=\frac{\bar{Y}}{\sum_{h=1}^{L} W_{h}\left\{\bar{Z}_{h}+\beta_{2 h}(z)\right\}}=\frac{\bar{Y}}{\bar{Z}_{1 h}}
$$

\section{EFFICIENCY COMPARISON}

Since we know that the variance of the usual unbiased estimator of the study variable $y$ in stratified random sampling is defined as

$$
V\left(\bar{y}_{s t}\right)=\sum_{h=1}^{L} W_{h}^{2} \gamma_{h} S_{y h}^{2}
$$

$\alpha_{\text {opt. }}=\frac{A}{B}$

From equations (7), (8), (9), (10), (11), (12), (16) and (18), where $A=2 \sum_{h=1}^{L} W_{h}^{2} \gamma_{h}\left[g_{h} R_{12} S_{y x h}+g_{h}^{2} R_{13}^{2} S_{z h}^{2}+g_{h} R_{13} S_{y z h}+g_{h}^{2} R_{12} R_{13} S_{z x h}^{\text {we }}\right]$ have

$$
B=\sum_{h=1}^{L} W_{h}^{2} \gamma_{h}\left[g_{h}^{2} R_{12}^{2} S_{x h}^{2}+2 g_{h}^{2} R_{13}^{2} S_{z h}^{2}+4 g_{h}^{2} R_{12} R_{13} S_{z x h}\right]
$$

(i) $\operatorname{MSE}\left(t_{s t}^{*}\right)<\operatorname{MSE}\left(\bar{y}_{s t}\right)$ iff

$$
\begin{gathered}
=\sum_{h=1}^{L} W_{h}^{2} \gamma_{h}\left[( 1 - \frac { A } { B } ) \left\{\left(1-\frac{A}{B}\right) g_{h}^{2} R_{13}^{2} S_{z h}^{2}\right.\right. \\
\left.+2 g_{h} R_{13} S_{y z h}-2 \frac{A}{B} g_{h}^{2} R_{12} R_{13} S_{x z h}\right\} \\
\left.-\frac{A}{B}\left\{2 g_{h} R_{12} S_{y x h}-\frac{A}{B} g_{h}^{2} R_{12}^{2} S_{x h}^{2}\right\}\right]<0
\end{gathered}
$$

or

$\min \cdot \operatorname{MSE}\left(t_{s t}^{*}\right)=M-P+Q+R+S-T$

(ii) $\operatorname{MSE}\left(t_{s t}^{*}\right)<\operatorname{MSE}\left(\bar{y}_{R C}\right)$ iff

where 


$$
\begin{aligned}
= & \sum_{h=1}^{L} W_{h}^{2} \gamma_{h}\left[( 1 - \frac { A } { B } ) \left\{\left(1-\frac{A}{B}\right) g_{h}^{2} R_{13}^{2} S_{z h}^{2}+2 g_{h} R_{13} S_{y z h}\right.\right. \\
& \left.-2 \frac{A}{B} g_{h}^{2} R_{12} R_{13} S_{x z h}\right\}-\frac{A}{B}\left\{2 g_{h} R_{12} S_{y x h}-\frac{A}{B} g_{h}^{2} R_{12}^{2} S_{x h}^{2}\right\} \\
& \left.+2 R_{1} S_{y x h}-R_{1}^{2} S_{x h}^{2}\right]<0
\end{aligned}
$$

(iii) $\operatorname{MSE}\left(t_{s t}^{*}\right)<\operatorname{MSE}\left(\bar{y}_{P C}\right)$ iff

$$
\begin{gathered}
=\sum_{h=1}^{L} W_{h}^{2} \gamma_{h}\left[\left(1-\frac{A}{B}\right) \int\left(1-\frac{A}{B}\right) g_{h}^{2} R_{13}^{2} S_{z h}^{2}+2 g_{h} R_{13} S_{y z h}\right. \\
\left.-2 \frac{A}{B} g_{h}^{2} R_{12} R_{13} S_{x z h}\right\}-\frac{A}{B}\left\{2 g_{h} R_{12} S_{y x h}-\frac{A}{B} g_{h}^{2} R_{12}^{2} S_{x h}^{2}\right\} \\
\left.-2 R_{2} S_{y z h}-R_{2}^{2} S_{z h}^{2}\right]<0
\end{gathered}
$$

(iv) $\quad \operatorname{MSE}\left(t_{s t}^{*}\right)<\operatorname{MSE}\left(\bar{y}_{R C}^{*}\right) \quad$ iff

$$
\begin{aligned}
= & \sum_{h=1}^{L} W_{h}^{2} \gamma_{h}\left[( 1 - \frac { A } { B } ) \left\{\left(1-\frac{A}{B}\right) g_{h}^{2} R_{13}^{2} S_{z h}^{2}+2 g_{h} R_{13} S_{y z h}\right.\right. \\
& \left.-2 \frac{A}{B} g_{h}^{2} R_{12} R_{13} S_{x z h}\right\}-\frac{A}{B}\left\{2 g_{h} R_{12} S_{y x h}-\frac{A}{B} g_{h}^{2} R_{12}^{2} S_{x h}^{2}\right\} \\
& \left.+2 R_{1} \mathrm{~g}_{\mathrm{h}} \mathrm{S}_{y x h}-R_{1}^{2} g_{h}^{2} S_{x h}^{2}\right]<0
\end{aligned}
$$

(v) $\operatorname{MSE}\left(t_{s t}^{*}\right)<\operatorname{MSE}\left(\bar{y}_{P C}^{*}\right) \quad$ iff

$$
\begin{aligned}
= & \sum_{h=1}^{L} W_{h}^{2} \gamma_{h}\left[( 1 - \frac { A } { B } ) \left\{\left(1-\frac{A}{B}\right) g_{h}^{2} R_{13}^{2} S_{z h}^{2}+2 g_{h} R_{13} S_{y z h}\right.\right. \\
& \left.-2 \frac{A}{B} g_{h}^{2} R_{12} R_{13} S_{x z h}\right\}-\frac{A}{B}\left\{2 g_{h} R_{12} S_{y x h}-\frac{A}{B} g_{h}^{2} R_{12}^{2} S_{x h}^{2}\right\} \\
& \left.-2 R_{2} \mathrm{~g}_{h} S_{y z h}-R_{2}^{2} g_{h}^{2} S_{z h}^{2}\right]<0
\end{aligned}
$$

(vi)

$$
\begin{gathered}
\operatorname{MSE}\left(t_{s t}^{*}\right)<\operatorname{MSE}\left(\bar{y}_{\mathrm{Re}}^{* s t}\right) \quad \text { iff } \\
=\sum_{h=1}^{L} W_{h}^{2} \gamma_{h}\left[( 1 - \frac { A } { B } ) \left\{\left(1-\frac{A}{B}\right) g_{h}^{2} R_{13}^{2} S_{z h}^{2}+2 g_{h} R_{13} S_{y z h}\right.\right. \\
\left.-2 \frac{A}{B} g_{h}^{2} R_{12} R_{13} S_{x z h}\right\}-\frac{A}{B}\left\{2 g_{h} R_{12} S_{y x h}-\frac{A}{B} g_{h}^{2} R_{12}^{2} S_{x h}^{2}\right\} \\
\left.+R_{1} \mathrm{~g}_{\mathrm{h}} \mathrm{S}_{y x h}-\frac{R_{1}^{2}}{4} g_{h}^{2} S_{x h}^{2}\right]<0
\end{gathered}
$$

\begin{tabular}{|c|c|c|c|c|}
\hline \multirow{7}{*}{$\begin{array}{l}\mathrm{N}=10 \\
\mathrm{n}=5\end{array}$} & $n_{1}=2$ & $n_{2}=3$ & $N_{1}=5$ & $N_{2}=5$ \\
\hline & $\begin{array}{l}\bar{Z}_{1}= \\
51.80\end{array}$ & $\begin{array}{l}\bar{Z}_{2}= \\
60.60\end{array}$ & $\begin{array}{c}\bar{X}_{1}= \\
214.4\end{array}$ & $\begin{array}{c}\bar{X}_{2}= \\
333.8\end{array}$ \\
\hline & $\begin{array}{c}\bar{Y}_{1}= \\
1925.8\end{array}$ & $\begin{array}{l}\bar{Y}_{2}= \\
3115.6\end{array}$ & $\begin{array}{l}S_{z_{1}}= \\
0.75\end{array}$ & $\begin{array}{l}S_{z_{2}}= \\
4.84\end{array}$ \\
\hline & $\begin{array}{l}S_{x_{1}}= \\
74.87\end{array}$ & $\begin{array}{l}S_{x_{2}}= \\
66.35\end{array}$ & $\begin{array}{l}S_{y_{1}}= \\
615.92\end{array}$ & $\begin{array}{c}S_{y_{2}}= \\
340.38\end{array}$ \\
\hline & $\begin{array}{l}S_{z x_{1}}= \\
38.08\end{array}$ & $\begin{array}{c}S_{z x_{2}}= \\
287.92\end{array}$ & $\begin{array}{l}S_{y z_{1}}= \\
-411.16\end{array}$ & $\begin{array}{l}S_{y z_{2}}= \\
-1536.24\end{array}$ \\
\hline & $\begin{array}{l}S_{y x_{1}}= \\
39360 . \\
68\end{array}$ & $\begin{array}{l}S_{y x_{2}}= \\
22356.5 \\
0\end{array}$ & $\begin{array}{l}C_{x_{1}}= \\
0.35\end{array}$ & $\begin{array}{l}C_{x_{2}} \\
=0.20\end{array}$ \\
\hline & $\begin{array}{l}C_{z_{1}}= \\
0.01\end{array}$ & $\begin{array}{l}C_{z_{2}}= \\
0.08\end{array}$ & & \\
\hline
\end{tabular}

$$
\begin{aligned}
& \text { (vii) } \operatorname{MSE}\left(t_{s t}^{*}\right)<\operatorname{MSE}\left(\bar{y}_{P e}^{* s t}\right) \quad \text { iff } \\
& =\sum_{h=1}^{L} W_{h}^{2} \gamma_{h}\left[( 1 - \frac { A } { B } ) \left\{\left(1-\frac{A}{B}\right) g_{h}^{2} R_{13}^{2} S_{z h}^{2}+2 g_{h} R_{13} S_{y z h}\right.\right. \\
& \left.-2 \frac{A}{B} g_{h}^{2} R_{12} R_{13} S_{x z h}\right\}-\frac{A}{B}\left\{2 g_{h} R_{12} S_{y x h}-\frac{A}{B} g_{h}^{2} R_{12}^{2} S_{x h}^{2}\right\} \\
& \left.-R_{2} \mathrm{~g}_{\mathrm{h}} \mathrm{S}_{y z h}-\frac{R_{2}^{2}}{4} g_{h}^{2} S_{z h}^{2}\right]<0
\end{aligned}
$$

From equations (19), (20), (21), (22), (23), (24) and (25), we obtained the conditions under which the suggested estimator performed well than other considered estimators.

\section{EMPIRICAL STUDY}

To judge the efficiency of the proposed estimator over other considered estimators, the following data sets are taken. The description of the population is given below:

Population I [Source: [19]]

y: Output

x: Fixed capital

z: Number of workers. Taking $n=5$

Population II [Source: [20]]

y: Productivity (MT/Hectare) 
$\mathrm{x}$ : Production in '000 Tons

z: Area in '000 Hectare. Taking $n=10$

\begin{tabular}{|c|c|c|c|c|}
\hline \multirow{12}{*}{$\mathrm{N}=20$} & $n_{1}=7$ & $n_{2}=3$ & $N_{1}=10$ & $N_{2}=10$ \\
\hline & $\bar{Z}_{1}=$ & $\bar{Z}_{2}=$ & $\bar{X}_{1}=$ & $\bar{X}_{2}=$ \\
\hline & 6.20 & 80.67 & 10.41 & 30.14 \\
\hline & $\bar{Y}_{1}=$ & $\bar{Y}_{2}=$ & $S_{z_{1}}=$ & $S_{z_{2}}=$ \\
\hline & 1.70 & 3.67 & 1.19 & 10.81 \\
\hline & $S_{x_{1}}=$ & $S_{x_{2}}=$ & $S_{y_{1}}=$ & $S_{y_{2}}=$ \\
\hline & 3.53 & 80.54 & 0.54 & 1.41 \\
\hline & $S_{z x_{1}}=$ & $S_{z x_{2}}=$ & $S_{y z_{1}}=$ & $S_{y z_{2}}=$ \\
\hline & 1.75 & 68.57 & -0.02 & -7.06 \\
\hline & $S_{y x_{1}}=$ & $S_{y x_{2}}=$ & $C_{x_{1}}=$ & $C_{x_{2}}=$ \\
\hline & 1.60 & 83.47 & 0.3390 & 2.6721 \\
\hline & $\begin{array}{c}C_{z_{1}}= \\
0.1919\end{array}$ & $\begin{array}{l}C_{z_{2}}= \\
0.1340\end{array}$ & & \\
\hline
\end{tabular}

For the purpose of the efficiency comparison of the proposed estimator, the percent relative efficiencies (PREs) of the estimators with respect to the usual unbiased estimator $\bar{y}_{s t}$ have been computed using the formula:

$\operatorname{PRE}\left(t, \bar{y}_{s t}\right)=\frac{\operatorname{MSE}\left(\bar{y}_{s t}\right)}{\operatorname{MSE}(t)} \times 100 ;$

where $t=\bar{y}_{s t}, \bar{y}_{R C}, \quad \bar{y}_{P C}, \bar{y}_{R C}^{*}, \quad \bar{y}_{P C}^{*}, \bar{y}_{\mathrm{Re}}^{* s t}, \bar{y}_{P \mathrm{e}}^{* s t}$ and $t_{s t}^{*}$

The findings are given in the Table 1 .

Table 1

Percent relative efficiencies of the estimators $\bar{y}_{s t}, \bar{y}_{R C}$, $\bar{y}_{P C}, \bar{y}_{R C}^{*}, \bar{y}_{P C}^{*}, \bar{y}_{\mathrm{Re}}^{* s t}, \bar{y}_{P \mathrm{e}}^{* s t}$ and $t_{s t}^{*}$ with respect to $\bar{y}_{s t}$

\begin{tabular}{|l|c|c|}
\hline Estimator & Population I & Population II \\
\hline $\bar{y}_{s t}$ & 100.00 & 100.00 \\
\hline $\bar{y}_{R C}$ & 651.0731 & 18383.618 \\
\hline $\bar{y}_{P C}$ & 245.3439 & 5390.124 \\
\hline
\end{tabular}

\begin{tabular}{|l|l|l|}
\hline $\bar{y}_{R C}^{*}$ & 235.4248 & 138.1285 \\
\hline $\bar{y}_{P C}^{*}$ & 237.2878 & 5035.331 \\
\hline $\bar{y}_{\mathrm{Re}}^{* s t}$ & 442.0247 & 16774.2264 \\
\hline $\bar{y}_{P \mathrm{e}}^{* s t}$ & 229.5866 & 5362.0528 \\
\hline$t_{s t}^{*}$ & 780.6235 & 22727.7675 \\
\hline
\end{tabular}

\section{CONCLUSION}

Section III reveals the conditions under which the suggested estimator has less MSE than other estimators. This means that the proposed estimator is more efficient than other considered estimators under certain limitations.

The table 1 represents that the suggested dual to ratio-cumproduct estimator has more percent relative efficiency as compared to the estimators $\bar{y}_{R C}, \bar{y}_{P C}, \bar{y}_{R C}^{*}, \bar{y}_{P C}^{*}, \bar{y}_{\mathrm{Re}}^{* s t}$, $\bar{y}_{P \mathrm{e}}^{* s t}$. Thus it can be concluded that if information on coefficient of variation of the auxiliary variable is available for each stratum then the suggested estimator performs well and more efficient than other considered estimators. Thus the suggested estimator can be recommended as an alternative use of the estimation of the population mean of the character understudy.

\section{REFERENCES}

[1] W.G. Cochran, "The estimation of the yields of cereal experiments by sampling for the ratio gain to total produce", Jour. Agri. Soc, 30, pp. 262-275, 1940.

[2] D.S. Robson, 1957, "Applications of multivariate polykays to the theory of unbiased ratio type estimation",

Jour. Amer. Statst. Assoc., 52, pp. 511-522, 1957.

[3] B.V.S. Sisodia and V.K. Dwivedi, "A modified ratio estimator using coefficient of variation of auxiliary variable", Journal Indian Society Agricultural Statistics, 33, pp. 13-18, 1981.

[4] H.P. Singh, R. Tailor, R. Tailor and M. Kakran, "An improved estimation of population mean using power transformation", Journal Indian Society Agricultural Statistics, 58, pp. 223-230, 2004.

[5] L.N. Upadhyaya and H.P. Singh, "Use of transformed auxiliary variable in estimating the finite population mean", Biometrical Journal, 41, pp. 627-636, 1999.

[6] L.N. Upadhyaya, H.P. Singh, S. Chatterjee and R. Yadav, " $A$ Generalized Family of Transformed Ratio-Product Estimators of Finite Population Mean in Sample Surveys", Model Assisted Statistics and Applications, 6, 2, pp. 137-150, 2011.

[7] L.N. Upadhyaya, H.P. Singh, S. Chatterjee and R. Yadav, "Improved Ratio and Product Exponential type Estimators for Finite Population Mean in Sample Surveys", Journal of Statistical Theory and Practice, 5, 2, pp. 285-302, 2011. 
[8] R. Yadav, L.N. Upadhyaya, H.P. Singh and S. Chatterjee, "Almost Unbiased Ratio and Product Type Exponential Estimators", Statistics in Transition, 13, 3, pp. 537-550, 2012.

[9] T. Srivenkataramana, "A dual of ratio estimator in sample surveys", Biometrika, 67, 1, pp. 199-204, 1980.

[10] S. Bandyopadhyay, "Improved ratio and product estimators", Sankhya: Indian J. Stat., 42, pp. 45-49, 1980.

[11] C. Kadilar and H. Cingi, "A new estimator in stratified random sampling”, Commun. Statist. Theor. Meth., 34, pp. 597-602, 2005.

[12] H.P. Singh and G.K. Vishwakarma, " A general procedure for estimating the population mean in stratified sampling using auxiliary information", Metron, LXVII, 1, pp. 47-65, 2010.

[13] R. Tailor and M. Chouhan, "Improved ratio-cum-product type exponential estimator of population mean in stratified random sampling", International Journal of Scientific Research in Mathematical and Statistical Sciences, 4, 3, pp. 19-29, 2017.

[14] R. Tailor, S. Chouhan, R. Tailor and N. Garg, "A ratio-cumproduct estimator of population mean in stratified random sampling using two auxiliary variables", Statistica, LXXII, 3, pp. 287-297, 2012.

[15] R. Yadav, L.N. Upadhyaya, H.P. Singh and S. Chatterjee, "Improved Ratio and Product Exponential type Estimators for Finite Population Mean in Stratified Random Sampling" Communications in Statistics: Theory \& Methods (Taylor \& Francis), 43, 15, pp. 3269-3285, 2014.

[16] M.H. Hansen, W.N. Hurwitz and M. Gurney, "Problem and methods of the sample survey of business", JASA, 41, pp. 174$189,1946$.

[17] K.S. Kushwaha, L.N. Upadhyaya and S.P. Dubey, "A dual to ratio estimator in stratified random sampling", Proc. Math. Soc., 6, pp. 11-15, 1990.

[18] R. Tailor, N.K. Jatwa, R. Tailor and N. Garg, "Dual to ratio and product type exponential estimators in stratified random sampling using two auxiliary variates", Journal of Reliability and Statistical Studies, 6, 2, pp. 115-126, 2013.

[19] M.N. Murthy, "Sampling theory and methods", Statistical Publishing Society, Calcutta, India 228, 1967.

[20] National Horticulture Board. Available at https://www.google.co.in/webhp?sourceid=chromeinstant\&ion $=1 \&$ espv= $=2 \&$ ie $=$ UTF-

$8 \# \mathrm{q}=$ National + Horticulture + Board $] \& *$

\section{AUTHOR PROFILE}

I have done my Ph.D. in Sample Surveys from IIT-ISM Dhanbad in 2012. Currently I am working as a Assistant Professor in Department of Statistics, Amity University, Noida, India. I have published 12 research papers in International Journals of repute. I have presented several research papers in National and International conferences.

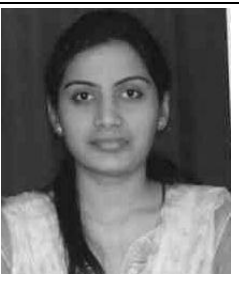
nd 